SUPPORTING INFORMATION

\title{
Oral Delivery of Parathyroid Hormone Using A Triple Padlock Nanocarrier for Osteoporosis via Enterohepatic Circulation Pathway
}

Jeong Man An,, ${ }^{\ddagger}$ S M Shatil Shahriar,, 1 Yong Hwa Hwang, ${ }^{2}$ Seung Rim Hwang, ${ }^{3}$ Dong Yun Lee, ${ }^{4,5}$ Sungpil Cho, ${ }^{*}, 6$ and Yong-kyu Lee ${ }^{*}, 1,2,6$

¥ These authors contributed equally to this work.

*Correspondence: Yong-kyu Lee (leeyk@ut.ac.kr) and Sungpil Cho (sungpilcho74@ut.ac.kr)

1Department of Chemical and Biological Engineering, Korea National University of Transportation, Chungju 27469, Republic of Korea

${ }^{2}$ KB Biomed Inc., Chungju 27469, Republic of Korea

${ }^{3}$ College of Pharmacy, Chosun University, Gwangju 61452, Republic of Korea

${ }^{4}$ Department of Bioengineering, College of Engineering, BK21 PLUS Future Biopharmaceutical Human Resources Training and Research Team, Institute of Nano Science \& Technology (INST), Hanyang University, Seoul 04763, Republic of Korea

Institute of Nano Science \& Technology (INST), Hanyang University, Seoul, 04763, Republic of Korea

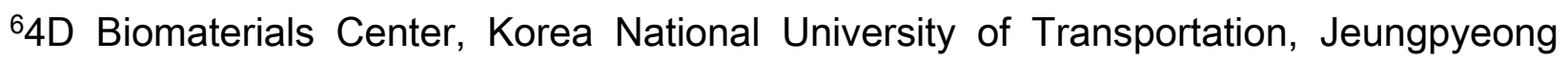
27909, Republic of Korea 


\section{SUPPLEMENTARY FIGURES}
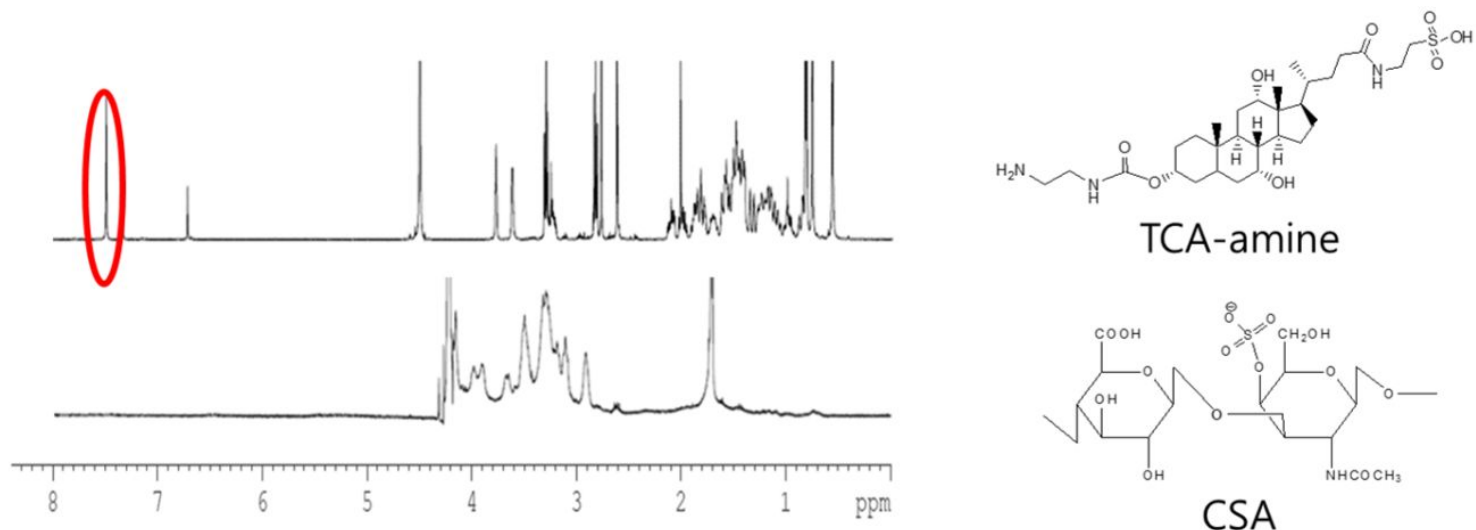

Figure S1. ${ }^{1} \mathrm{H}-\mathrm{NMR}$ spectra of CSA and TCA-amine. The relevant proton signals are in linear and circular boxes with red color.

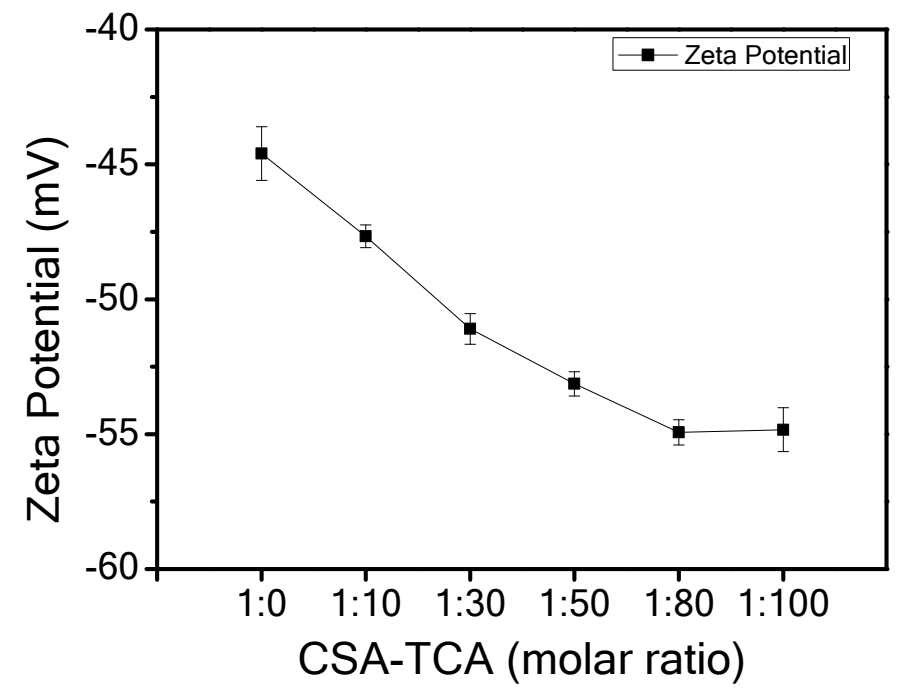

Figure S2. The surface charges of TCSA. The zeta potential values of TCSA were measured to optimize the conjugation ratio between CSA and TCA-NH $\mathrm{H}_{2}$. As the amount 


\section{SUPPORTING INFORMATION}

of conjugated TCA increases, the value of the zeta potential decreases. Data were presented as mean $\pm S D, n=3$.

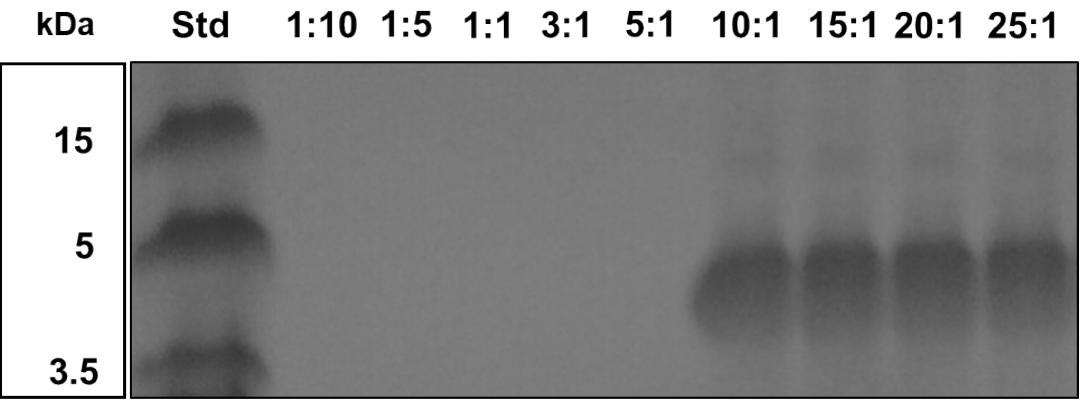

Figure S3. Measurement of loading efficiency of rhPTH (1-34) to TCSA by SDS-PAGE. The SDS-PAGE exhibited that 5:1 molar ratio is the optimum complexing ratio for TCSA : rhPTH, in which 5 molar ratios of TCSA could load $100 \%$ of 1 molar equivalent rhPTH $(1-34)$.

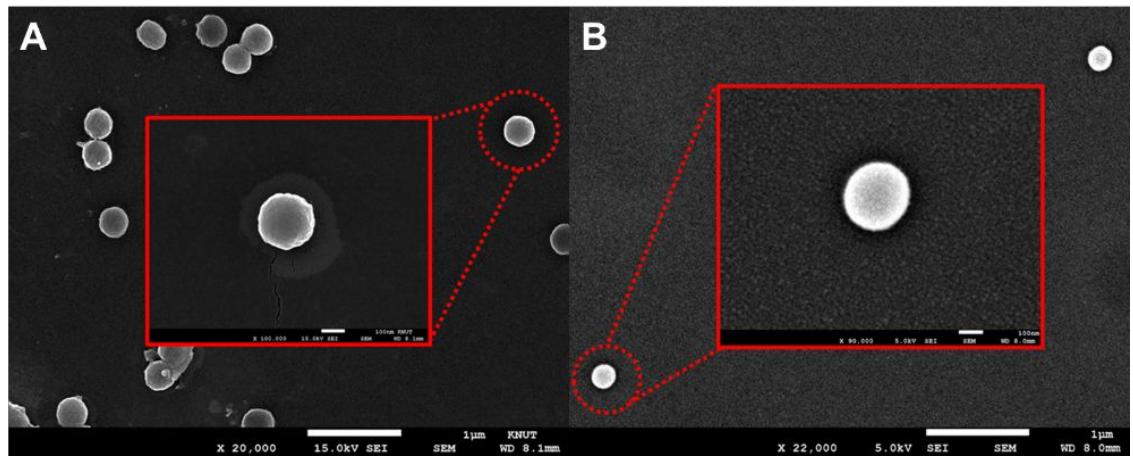

Figure S4. Representative electron microscopic images from FE-SEM displaying morphologies of (A) TCSA/rhPTH and (B) CSA/rhPTH. 


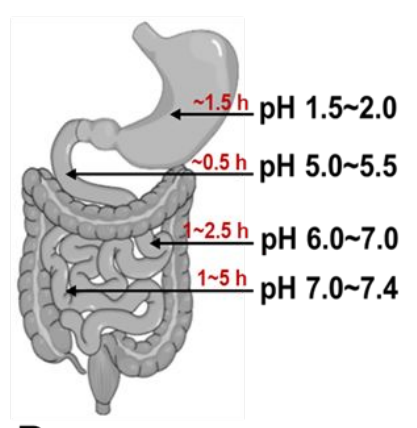

B

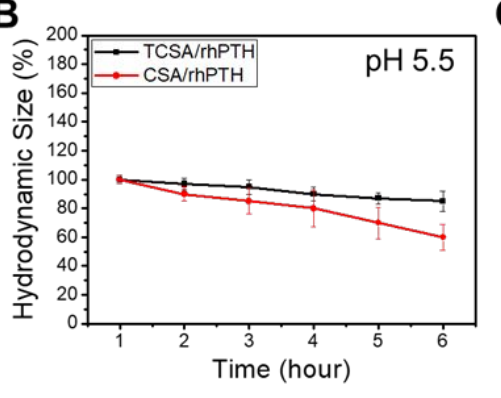

A

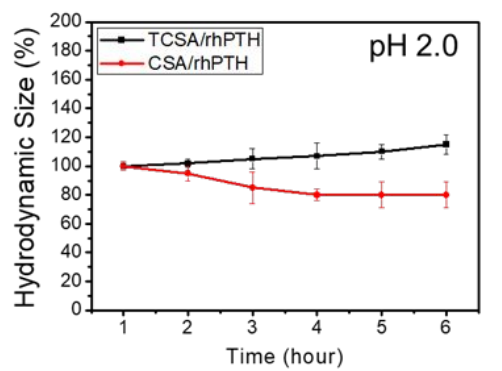

C

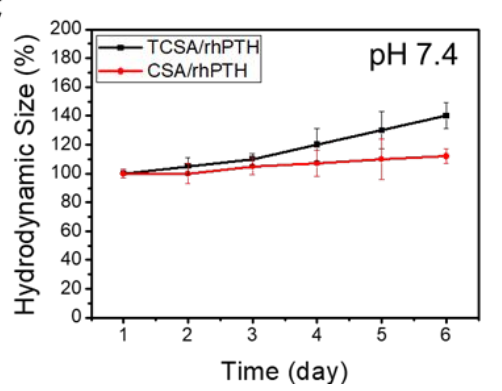

Figure S5. Changes in the particle size of TCSA/rhPTH and CSA/rhPTH NPs at the various gastrointestinal $(\mathrm{GI}) \mathrm{pH}$ environments. (A-C) Stability analysis of TCSA/rhPTH and $\mathrm{CSA} / \mathrm{rhPTH}$ at $\mathrm{pH} 2,5.5$ and 7.4 (mean $\pm \mathrm{SD}, \mathrm{n}=3$ ).

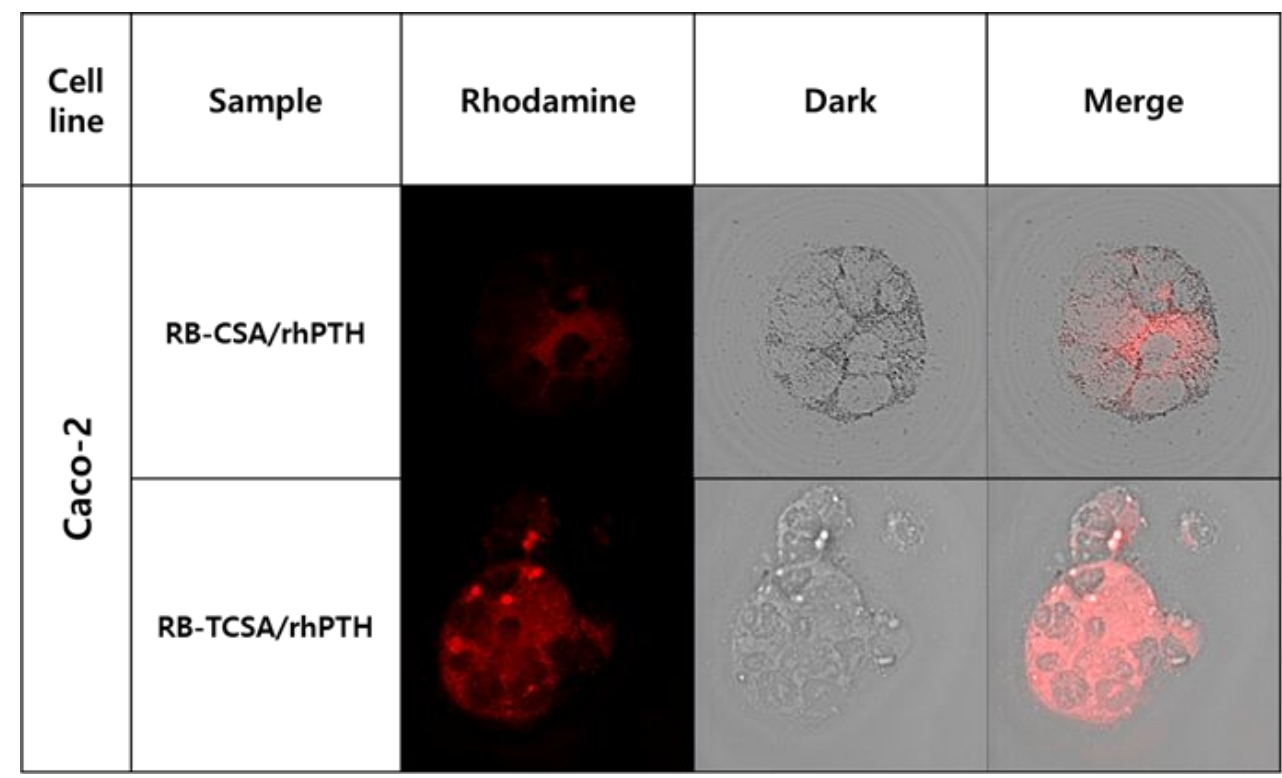


Figure S6. Uptake of RB-TCSA/rhPTH and RB-CSA/rhPTH by monolayered Caco-2 (human intestinal epithelial cell line) cell line, bile acid receptor-positive cells.

\begin{tabular}{|c|c|c|c|c|}
\hline $\begin{array}{l}\text { Cell } \\
\text { line }\end{array}$ & Sample & Rhodamine & Dark & Merge \\
\hline \multirow{2}{*}{$\begin{array}{l}\text { ㅇํㅁ } \\
\frac{0}{1}\end{array}$} & RB-CSA/rhPTH & & & \\
\hline & RB-TCSA/rhPTH & & & \\
\hline
\end{tabular}

Figure S7. Uptake of rhodamine B isothiocyanate (RB)-conjugated TCSA/rhPTH and CSA/rhPTH by HepG2 (human liver epithelial cell line)

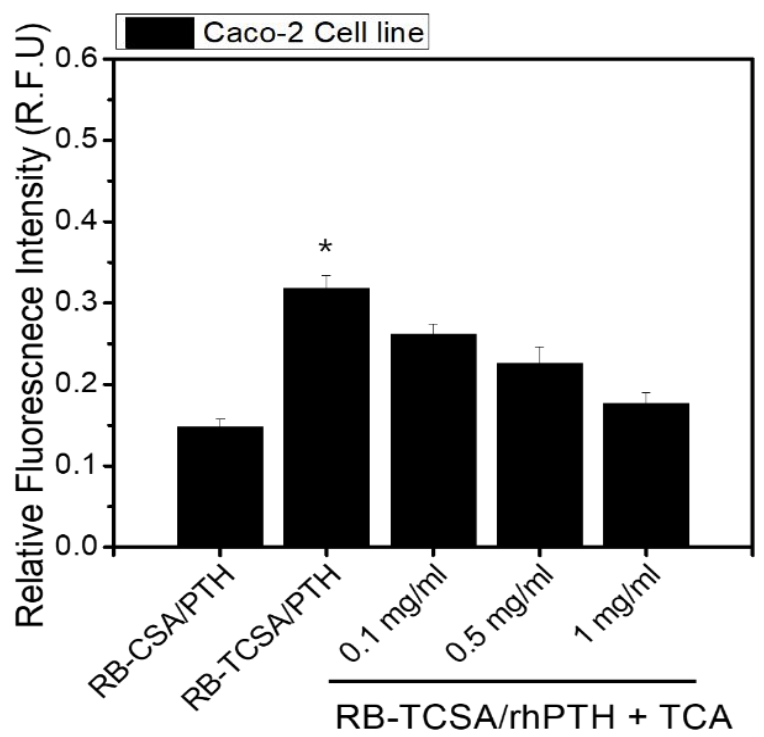


Figure S8. Competitive cellular uptake assay of TCSA/rhPTH in Caco-2 cell line pretreated with different concentrations of TCA (mean $\pm \mathrm{SD} ; \mathrm{n}=5 ;{ }^{*} p<0.05$ ).

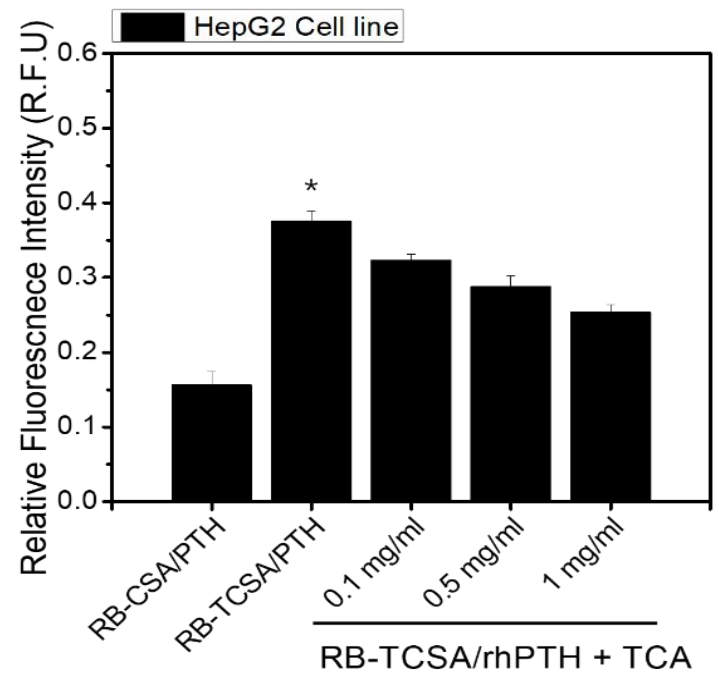

Figure S9. Competitive cellular uptake assay of RB-TCSA/rhPTH in HepG-2 cell line pretreated with different concentrations of TCA (mean $\pm \mathrm{SD}, \mathrm{n}=5$; ${ }^{*} p<0.05$ ).

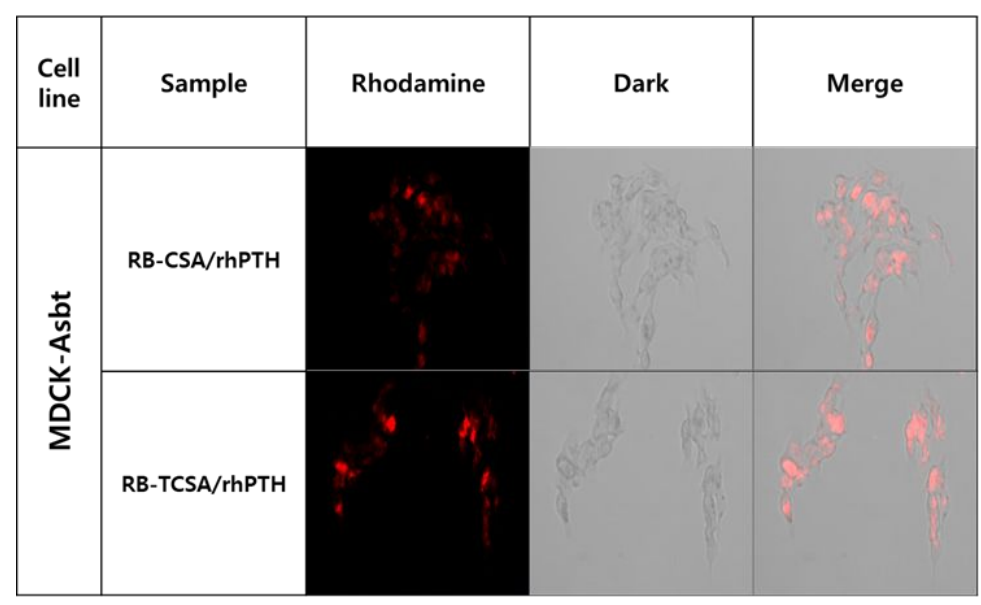

Figure S10. Confocal images exhibiting cellular uptake of RB-TCSA/rhPTH and RBCSA/rhPTH into the ASBT-expressed MDCK (MDCK-ASBT) cell line. 


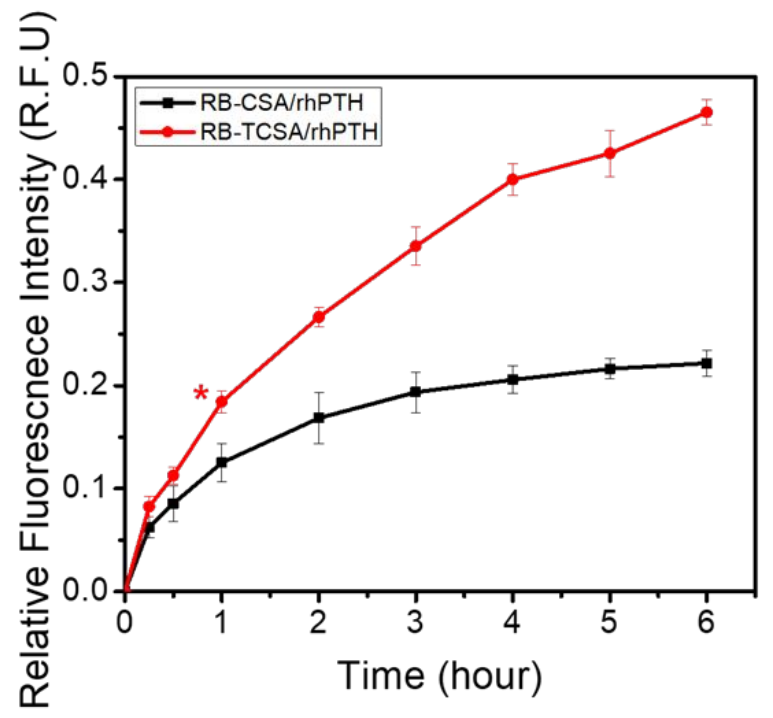

Figure S11. Quantitative analysis of cellular uptake between RB-TCSA/rhPTH and RB$\mathrm{CSA} / \mathrm{rhPTH}$ in both MDCK-ASBT cell (mean \pm S.D., $\mathrm{n}=5$; * $p<0.05$ ).

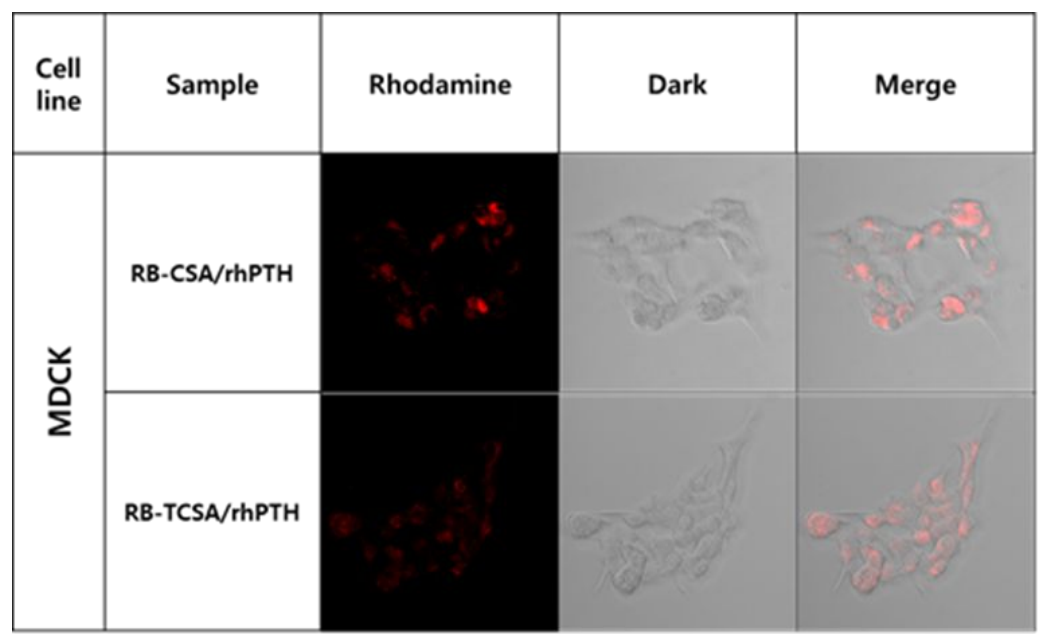

Figure S12. Confocal images exhibiting cellular uptake of RB-TCSA/rhPTH and RB$\mathrm{CSA} / \mathrm{rhPTH}$ into the MDCK cell line. 


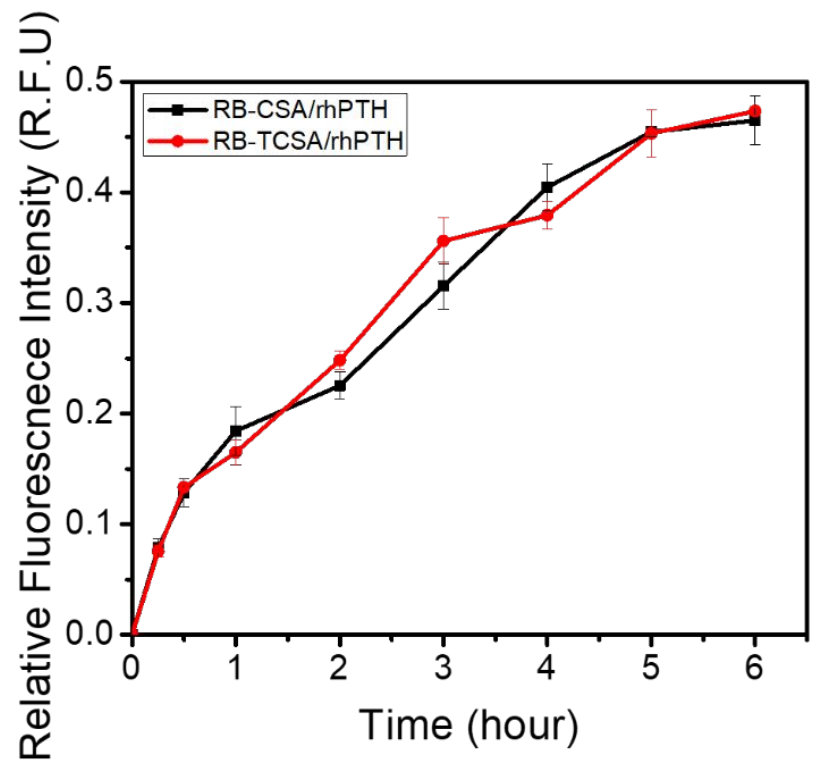

Figure S13. Quantitative analysis of cellular uptake between RB-TCSA/rhPTH and RB$\mathrm{CSA} / \mathrm{rhPTH}$ in MDCK cells (mean $\pm \mathrm{SD}, \mathrm{n}=5$ ).

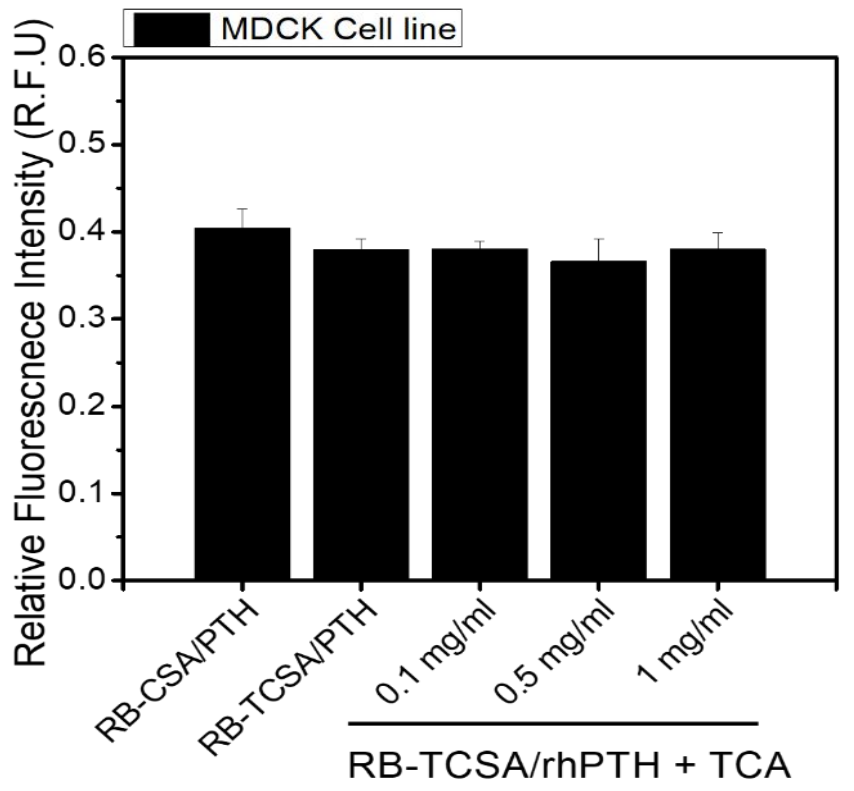

Figure S14. Competitive cellular uptake assay to evaluate the role of TCA in cellular uptake of RB-TCSA/rhPTH into MDCK cell line after TCA pretreatment (mean \pm SD; $n=$ $5)$. 


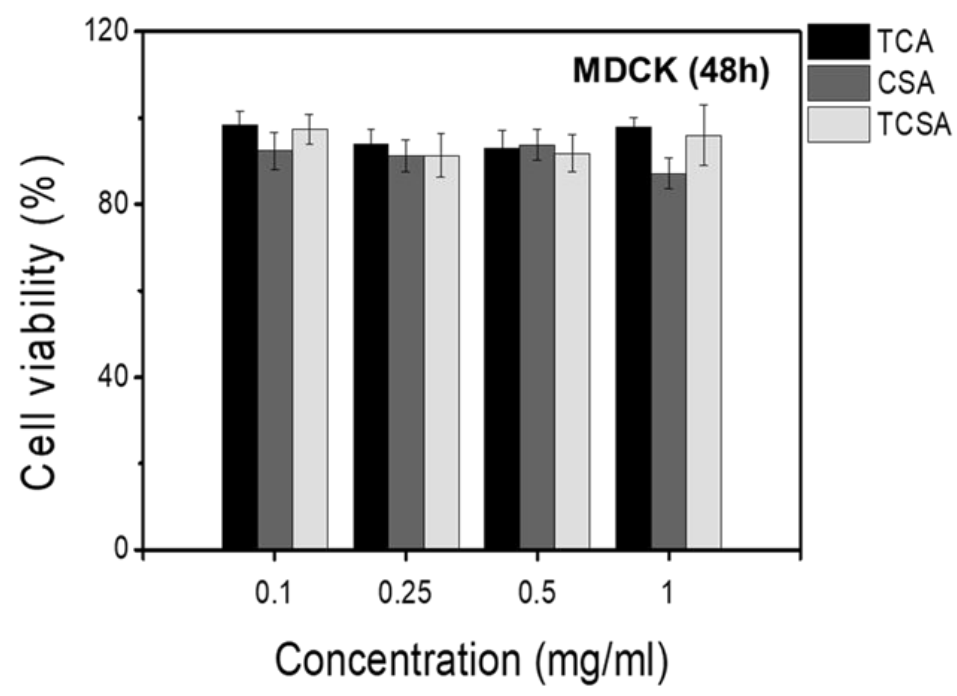

Figure S15. Cellular cytotoxicity assay of TCA, CSA, and TCSA to the MDCK cell line after 48 hours of incubation (mean $\pm S D ; n=5$ ).

A

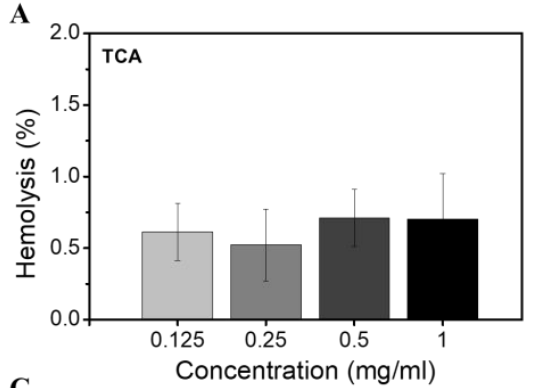

C

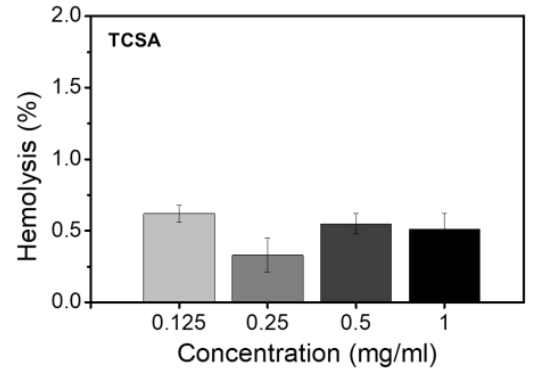

B

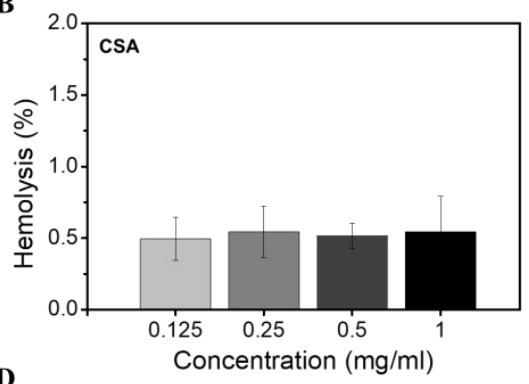

D

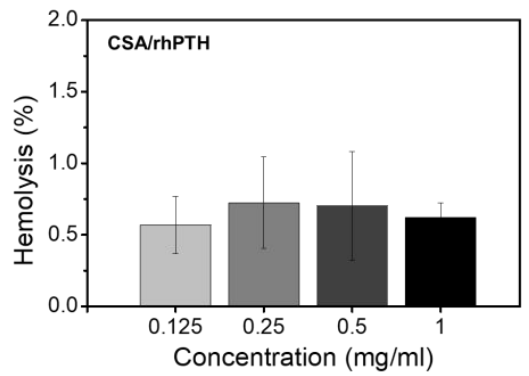

Figure S16. Hemocompatibility assay for TCA (A), CSA (B), TCSA (C) and CSA/rhPTH (D). Data were presented as (mean $\pm S D ; n=5)$ 

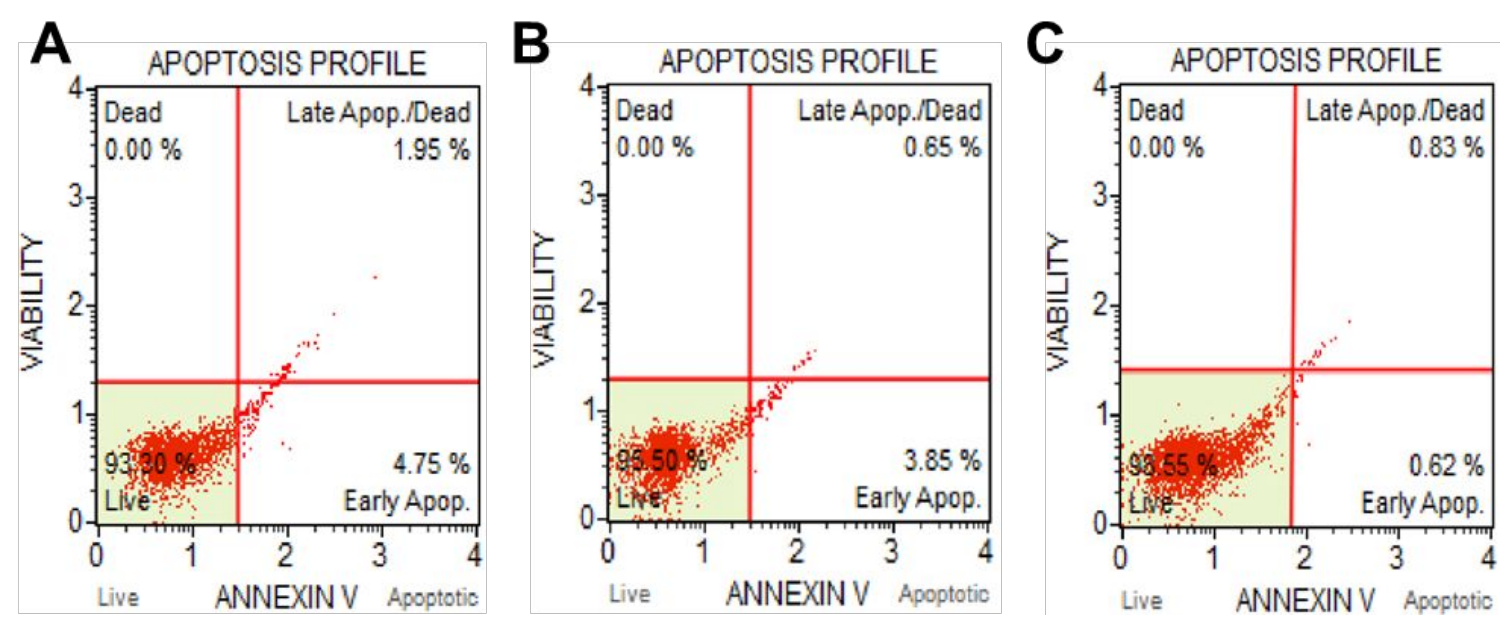

Figure S17. Annexin $\mathrm{V}$ apoptosis assay was used to study the programmed cell death in the (A) MDCK, (B) HepG2, and (C) Caco-2 cell lines incubated with the highest concentration of TCSA/rhPTH $(5 \mathrm{mg} / \mathrm{ml})$.

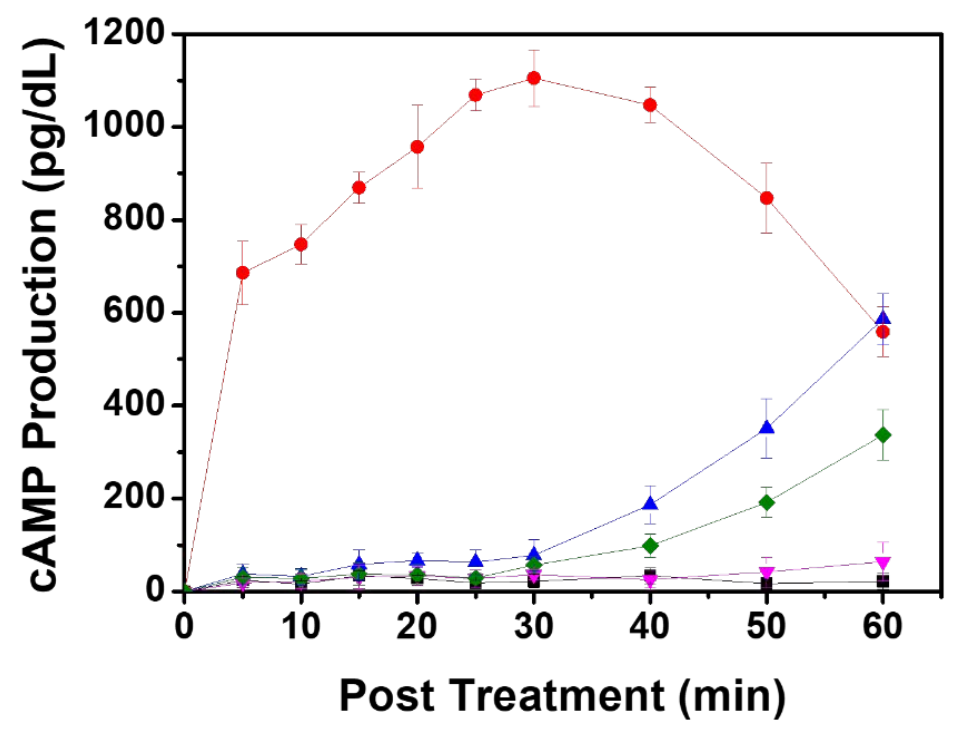

Figure S18. Intracellular cAMP levels in mouse osteoblast cells treated with $\mathrm{rhPTH}$ alone or in combination with CSA or TCSA. Red: $\mathrm{rhPTH}(4.05 \mathrm{ng} / \mathrm{mL})$; Blue: CSA/rhPTH (4.05 ng/mL); Green: TCSA/rhPTH (4.05 ng/mL); Pink: TCSA/rhPTH (1.45 ng/mL); Black: PBS. Since the stimulative effect of rhPTH on cAMP production in cells is very rapid, naked rhPTH produces the maximum concentration of cAMP within 30 minutes 
and then decreases over time. However, these stimulatory effects of CSA/rhPTH and TCSA/rhPTH are relatively slow compared to naked rhPTH which is consistent with their release profile (Figure 1D-E). Data were presented as mean $\pm S D, n=3$.
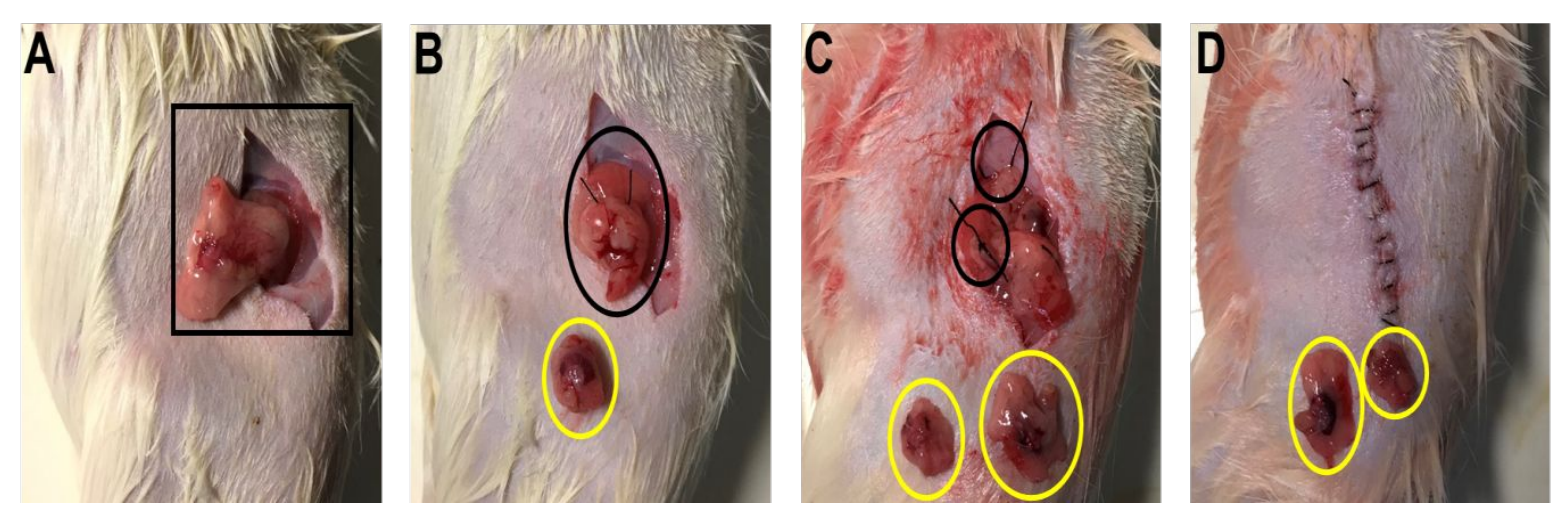

Figure S19. Development of an OVX rat model by performing bilateral ovariectomy of 12-week-old female SD rats. (A)-(D) surgical procedures to remove both ovaries from the rat.

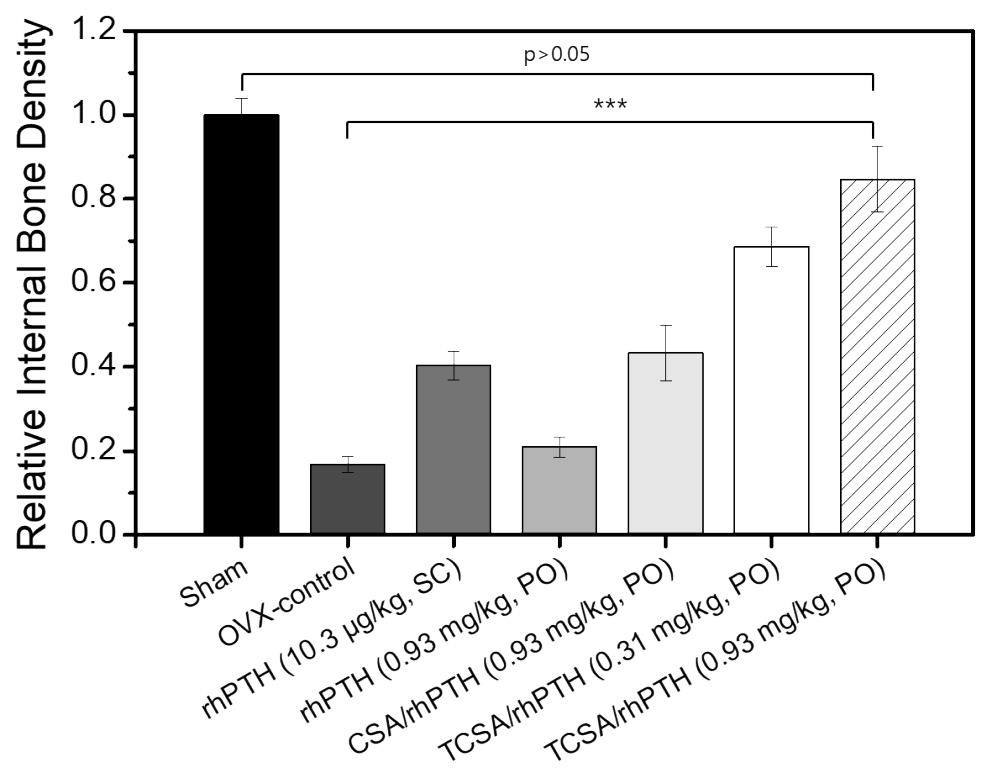


Figure S20. Micro-CT based quantification of internal bone mass. OVX-rats were orally administered with various dosages of rhPTH (either alone or in combination with CSA or TCSA) or PBS and their bone mass was compared with the sham-control or S.C. dose of rhPTH. Data were presented as mean \pm S.D. ${ }^{* * *} p<0.01$.

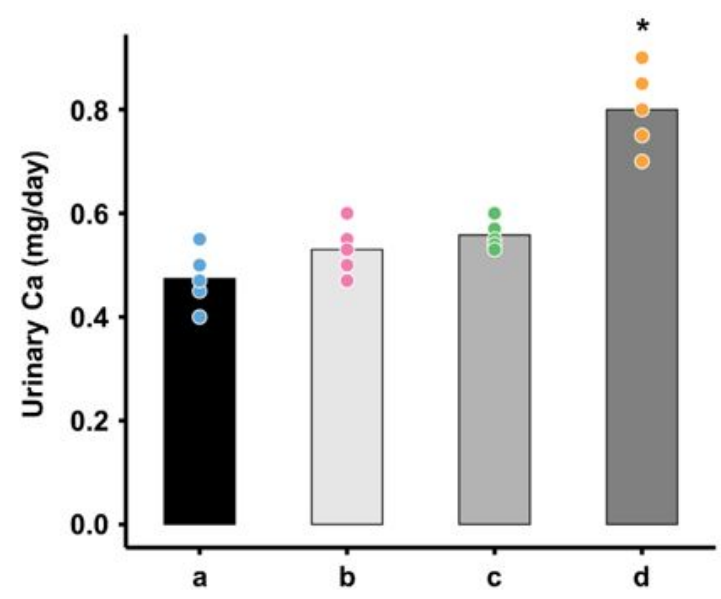

Figure S21. The effect of TCSA/rhPTH in urinary calcium excretion. (Here, the alphabetical order from a-d represents various treatment groups, such as, a, shamcontrol; b, subcutaneously delivery of $10.34 \mu \mathrm{g} / \mathrm{kg} \mathrm{rhPTH} \mathrm{(1-34);} \mathrm{c,} \mathrm{oral} \mathrm{delivery} \mathrm{of} 0.93$ $\mathrm{mg} / \mathrm{kg} \mathrm{TCSA} / \mathrm{rhPTH}$; d, PBS. Data were presented as mean $\pm \mathrm{SD}, \mathrm{n}=5$; * indicates $\mathrm{p}$ value $<0.01)$. 


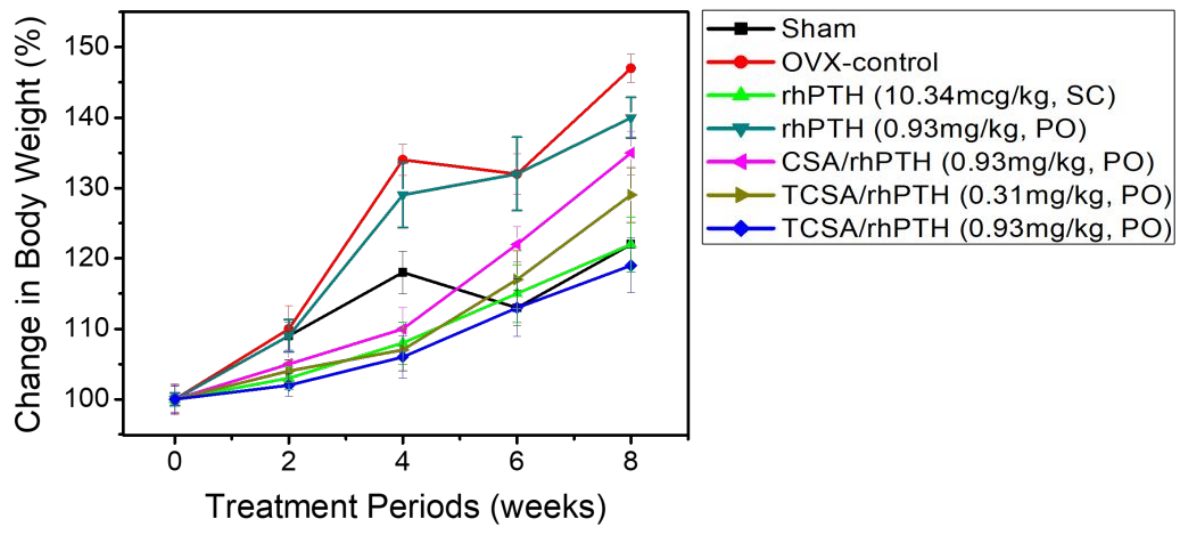

Figure S22. The line graph indicates how the body weight of the treated rats changed over time in comparison with normal and experimental control groups. Data are presented as mean $\pm S D, n=7$.
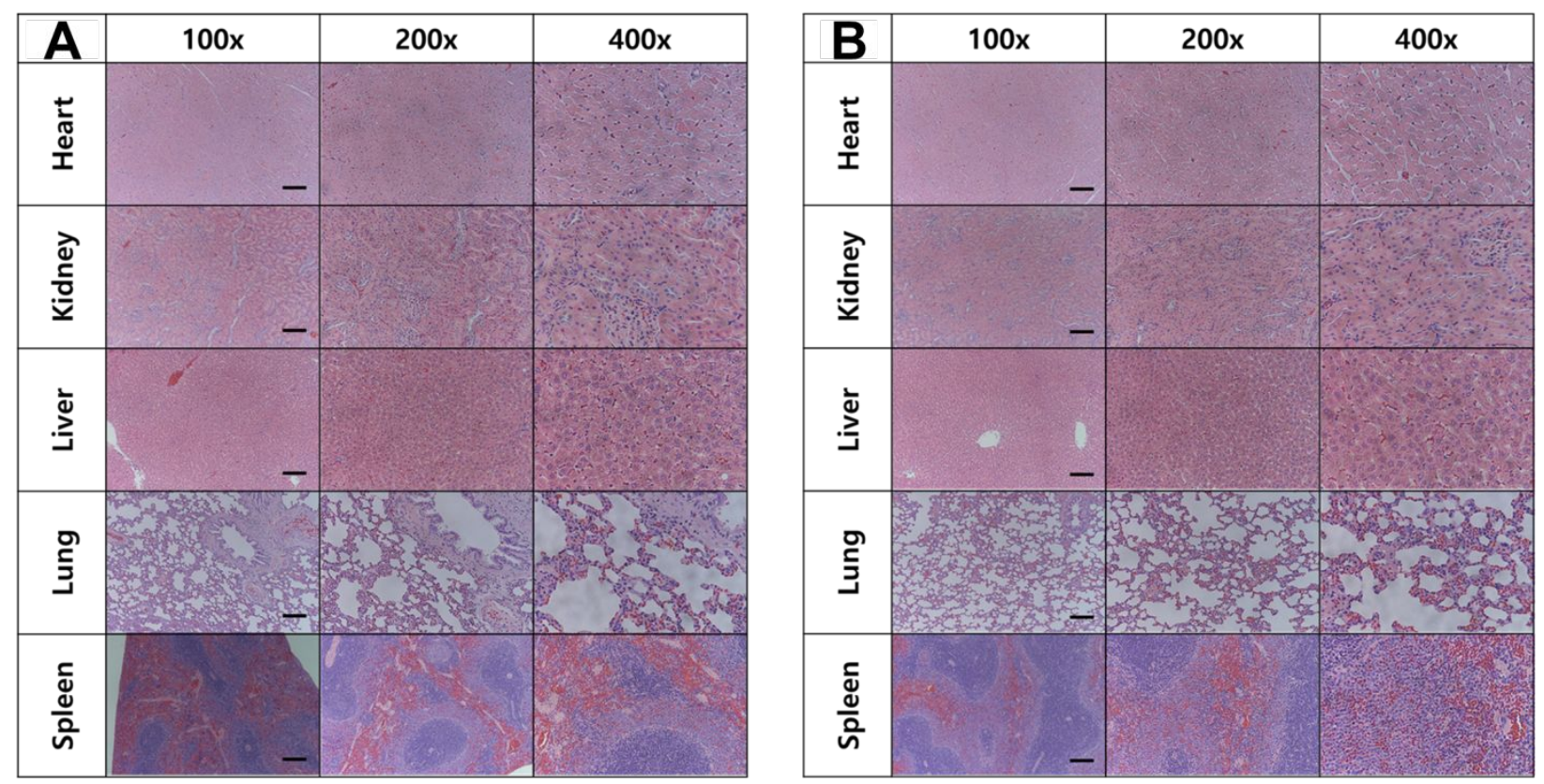

Figure S23. (A-B) Immunohistopathology of various tissues (heart, kidney, liver, lung, and spleen) of normal female SD rats $(n=3-5)(B)$, compared to OVX-rats $(C)$ at day 61 after once-daily oral administration of TCSA/rhPTH (scale $=100 \mu \mathrm{m})$. 


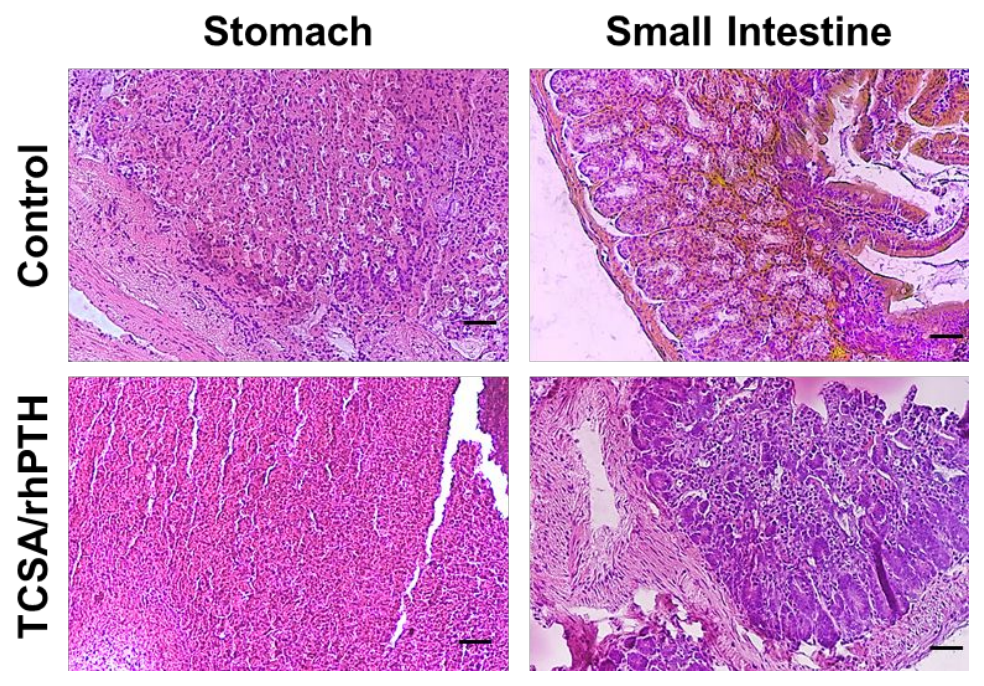

Figure S24. Gastrointestinal irritation assay with hematoxylin and eosin staining of stomach and small intestine after oral administration of TCSA/rhPTH. Each scale bar represents $100 \mu \mathrm{m}$ of tissue.
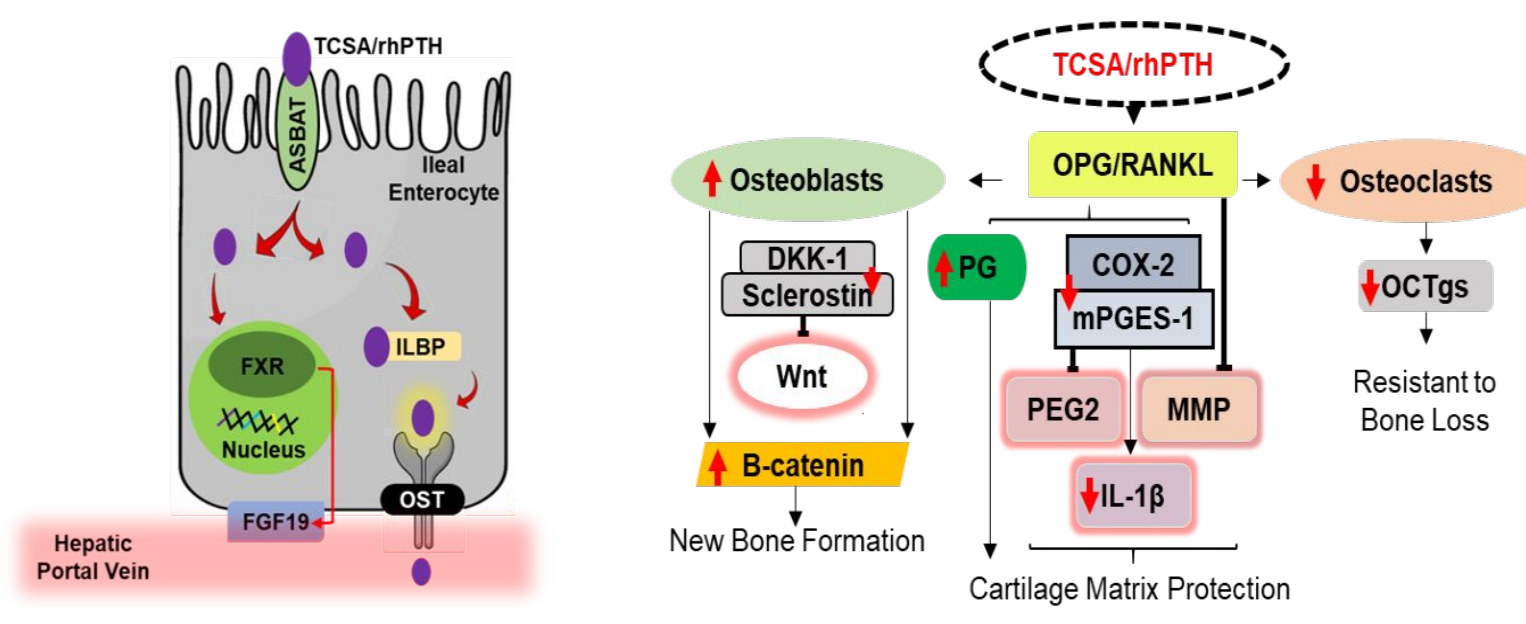

Figure S25. Intestinal epithelial cell bile acid sensing (left panel) and triple-padlock antiosteoporotic mechanism of TCSA/rhPTH (right panel). 
SUPPORTING INFORMATION

\section{SUPPLEMENTARY TABLES}

Table S1. Conjugation of ethylenediamine (EDA) to 4-nitrophenyl chloroformateconjugated taurocholic acid (TCA-NPC).

\begin{tabular}{|ccc|}
\hline Molar Ratio & TCA-NPC & EDA \\
\hline $1: 10$ & $500 \mathrm{mg}$ & $44.1 \mathrm{ug}$ \\
\hline $1: 30$ & $500 \mathrm{mg}$ & $132.4 \mathrm{ug}$ \\
\hline
\end{tabular}


SUPPORTING INFORMATION

$1: 50 \quad 500 \mathrm{mg} \quad 220.7 \mathrm{ug}$

Table S2. The dynamic light scattering (DLS) and surface charge (zeta potential) of TCA-amine after conjugations between EDA and TCA-NPC (mean \pm SD, $n=3$ ).

\begin{tabular}{|ccc|}
\hline Molar Ratio & DLS (nm in diameter) & Zeta Potential (mV) \\
\hline $1: 1$ & $35 \pm 8$ & $-19 \pm 5$ \\
\hline $1: 3$ & $46 \pm 2$ & $-17 \pm 1$ \\
\hline $1: 5$ & $49 \pm 4$ & $-15 \pm 2$ \\
\hline $1: 10$ & $55 \pm 3$ & $-11 \pm 2$ \\
\hline $1: 15$ & $54 \pm 5$ & $-10 \pm 4$ \\
\hline
\end{tabular}

Table S3. Conjugation of the various molar ratio of TCA-amine to chondroitin sulfate A (CSA).

\begin{tabular}{|ccc|}
\hline Molar Ratio & CSA & $\mathrm{NH}_{2}$-conjugated TCA \\
\hline $1: 10$ & $100 \mathrm{mg}$ & $21.5 \mathrm{ug}$ \\
\hline $1: 30$ & $100 \mathrm{mg}$ & $64.5 \mathrm{ug}$ \\
\hline $1: 50$ & $100 \mathrm{mg}$ & $107.5 \mathrm{ug}$ \\
\hline $1: 80$ & $100 \mathrm{mg}$ & $172 \mathrm{ug}$ \\
\hline $1: 100$ & $100 \mathrm{mg}$ & $215 \mathrm{ug}$ \\
\hline
\end{tabular}


SUPPORTING INFORMATION

Table S4. The surface charge of TCSA conjugates (mean $\pm S D, n=3$ ).

\begin{tabular}{|c|c|}
\hline Sample & Zeta Potential (mV) \\
\hline TCA-NH 2 & $-44.6 \pm 0.9$ \\
\hline CSA : TCA-NH 2 (TCSA, 1:10) & $-47.7 \pm 0.4$ \\
\hline TCSA (1:30) & $-51.1 \pm 0.6$ \\
\hline TCSA (1:50) & $-53.1 \pm 0.4$ \\
\hline TCSA (1:80) & $-54.9 \pm 0.5$ \\
\hline TCSA (1:100) & $-54.8 \pm 0.8$ \\
\hline
\end{tabular}

Table S5. TNBSA and zeta potential analysis of TCSA (1:80) to quantify the actual conjugation ratio between CSA and TCA (mean \pm SD, $n=3$ ).

\begin{tabular}{|cccc|} 
Sample Name & $\begin{array}{c}\text { Feed Ratio } \\
(\text { CSA : TCA) }\end{array}$ & $\begin{array}{c}\text { Conjugation Ratio } \\
(\text { CSA : TCA })\end{array}$ & $\begin{array}{c}\text { Zeta Potential } \\
(\mathbf{m V})\end{array}$ \\
\hline CSA & $1: 0$ & None & $-44.6 \pm 0.9$ \\
\hline TCA & $0: 1$ & None & $-21.3 \pm 0.7$ \\
\hline TCSA & $1: 80$ & $1: 44 \pm 0.8$ & $-54.9 \pm 0.5$ \\
\hline
\end{tabular}

Table S6. The nanoparticle size and PDI of human recombinant parathyroid hormone (rhPTH), TCSA, TCSA/rhPTH (5:1) and CSA/rhPTH (10:1) were measured by DLS whereas the surface charges of all nanoparticles were measured by zeta potential (mean $\pm \mathrm{SD}, \mathrm{n}=5$ ). 
SUPPORTING INFORMATION

\begin{tabular}{|lccc|}
\hline Nanoparticle & Size $(\mathrm{nm})$ & PDI & Surface Charge $(\mathrm{mV})$ \\
\hline rhPTH & $83.7 \pm 6.6$ & $0.45 \pm 0.017$ & $44.7 \pm 2.5$ \\
\hline TCSA & $257.2 \pm 9.01$ & $0.21 \pm 0.047$ & $-54.9 \pm 0.5$ \\
\hline TCSA/rhPTH & $230 \pm 6$ & $0.38 \pm 0.034$ & $-5.1 \pm 0.95$ \\
\hline CSA/rhPTH & $251 \pm 17$ & $0.33 \pm .013$ & $-7.5 \pm 2.03$ \\
\hline
\end{tabular}

Table S7. Early and late programmed cell death assay in vitro was performed using MUSE cell analyzer (Millipore Corporation, USA) to evaluate the level apoptosis in MDCK (upper table), HepG2 (middle table) and Caco-2 (lower table) cell lines treated with the $5 \mathrm{mg} / \mathrm{ml}$ concentration of TCSA $/ \mathrm{rhPTH}$.

\begin{tabular}{|c|c|c|}
\hline MDCK Cell & Cell Concentration (cells/ml) & $\%$ Gated \\
\hline Live (LL) & $1.63 E+06$ & $93.30 \%$ \\
\hline Early Apoptotic (LR) & $8.13 E+04$ & $4.75 \%$ \\
\hline $\begin{array}{lll}\text { Late } & \text { Apop.l Dead } \\
\text { (UR) } & & \end{array}$ & $3.41 \mathrm{E}+04$ & $1.95 \%$ \\
\hline Debris (UL) & $0.00 \mathrm{E}+00$ & $0.00 \%$ \\
\hline Total Apoptotic & $1.17 \mathrm{E}+05$ & $6.70 \%$ \\
\hline
\end{tabular}


SUPPORTING INFORMATION

\begin{tabular}{|lcc|}
\hline Live (LL) & $2.60 \mathrm{E}+06$ & $95.50 \%$ \\
\hline Early Apoptotic (LR) & $1.05 \mathrm{E}+05$ & $3.83 \%$ \\
\hline $\begin{array}{l}\text { Late Apop./ Dead } \\
(\mathrm{UR})\end{array}$ & $1.77 \mathrm{E}+04$ & $0.65 \%$ \\
\hline Debris (UL) & $0.00 \mathrm{E}+00$ & $0.00 \%$ \\
\hline Total Apoptotic & $1.22 \mathrm{E}+05$ & $4.50 \%$ \\
\hline
\end{tabular}

\begin{tabular}{|lcc|}
\hline \multicolumn{1}{|c}{ Caco-2 Cell } & Cell Concentration (cells/ml) & \% Gated \\
\hline Live (LL) & $3.81 \mathrm{E}+06$ & $98.55 \%$ \\
\hline Early Apoptotic (LR) & $2.41 \mathrm{E}+04$ & $0.62 \%$ \\
\hline Late Apop./ Dead (UR) & $3.21 \mathrm{E}+04$ & $0.83 \%$ \\
\hline Debris (UL) & $0.00 \mathrm{E}+00$ & $0.00 \%$ \\
\hline Total Apoptotic & $5.62 \mathrm{E}+04$ & $1.45 \%$ \\
\hline
\end{tabular}

